\title{
Lonely at the top and stuck in the middle? The ongoing challenge of using cost-effectiveness information in priority setting
}

Comment on "Use of cost-effectiveness data in priority setting decisions: experiences from the national guidelines for heart diseases in Sweden"

\author{
Iestyn Williams ${ }^{1^{*}}$, Stirling Bryan ${ }^{2}$
}

\section{Abstract}

The topic of how cost-effectiveness information informs priority setting in healthcare remains important to both policy and practice. This commentary considers the study carried out by Eckard and colleagues in Sweden. In it we distinguish between the conditions at national and local levels and put forward some recommendations for research into local priority setting in particular.

Keywords: Priority Setting, Economic Evaluation, Decision-making, Healthcare, Technology Coverage Copyright: @ 2015 by Kerman University of Medical Sciences

Citation: Williams I, Bryan S. Lonely at the top and stuck in the middle? The ongoing challenge of using cost-effectiveness information in priority setting: Comment on "Use of cost-effectiveness data in priority setting decisions: experiences from the national guidelines for heart diseases in Sweden". Int J Health Policy Manag 2015; 4: 185-187. doi: 10.15171/ijhpm.2015.32

\section{Article History:}

Received: 23 January 2015

Accepted: 11 February 2015

ePublished: 15 February 2015
$\mathrm{W}$ ithin these pages Eckard and colleagues (1) contribute to the growing evidence base on usage of economic evaluation in priority setting, adding a uniquely Swedish dimension to the qualitative explorations of the last few years. Their findings and conclusions appear to confirm the divergence in experience between decisionmaking tiers: whilst on the one hand national guidance producing bodies seek to routinely incorporate economic evaluation into their activities and indeed many are now formally mandated to do so, on the other hand local decisionmakers are disinclined or else unable to do the same (2-4).

However, Eckard and colleagues (1) also remind us that not all is rosy at the national level. Despite a positive headline finding, their study uncovers persistent deficits in the quality and relevance of the economic evidence obtained, as well as the expertise required for its interpretation and critique. Furthermore, their study hints at some unresolved questions of ethics and efficiency. For example, there are equity concerns raised when a decision-making body makes only selective recourse to cost-effectiveness as a criterion when due process might imply that the same criteria are applied in each instance. The authors note that only in cases where evidence of improved clinical effectiveness is lacking is the cost-effectiveness 'hurdle' invoked. This prioritising of clinical benefit over relatively cost-effectiveness arguably raises further concerns regarding efficiency: for example, in a context of economic constraint would this approach preclude adoption of new treatments with marginal clinical inferiority but vastly reduced costs? And if so, does this not lead to a unnecessarily high opportunity costs?

These limitations and challenges aside, there is clearly a gap between the experience of national bodies - in this case the Swedish National Board of Health and Welfare - and the meso level function, however this latter is configured within country systems, and we believe this divergence is worthy of further consideration.

The concern in this commentary is not to advocate for either an increased or reduced role for economic analysis in coverage decision-making but rather to examine current patterns in usage and the apparent divergence between national and local tiers. Indeed we believe the language of 'barriers' to greater usage is loaded with normative intent and therefore should be avoided (5). Eckard and colleagues make effective use of the accessibility-acceptability framework to categorise impediments to an economics-informed approach $(6,7)$. In this context accessibility relates to the shortage of relevant analyses as well as lack of expertise in its interpretation and critique. Whilst well-resourced national bodies can take steps to address such shortfalls, local decision-makers rarely have the resources to do so. Furthermore, at the local level the complex and interactive nature of the decision-making environment makes economic analysis less acceptable to those involved and in this respect, little has changed since the early work of Rudolf Klein (8) who observed:

'Priority setting is a complex interaction of multiple decisions at various levels in the organisation and constrained by history. There is no self-evident set of ethical principles or analytical tools to determine what decisions we should take at various levels, nor is there an obvious or easy way to resolve the clash of claims on resources.'

Far from reflecting a linear model of decision-making, much local priority setting is subject to multiple influencing

Full list of authors' affiliations is available at the end of the article. 
factors including political considerations, administrative imperatives, distributional concerns, societal opinion and so on. Perhaps unsurprisingly therefore decision-makers perceive recommendations from economic analyses to be difficult to implement. For example, budget holders operating within short-term budgeting cycles may be under pressure to contain cost over and above promoting efficiency or may experience difficulties redirecting resources across inflexible financial structures.

It is important to note that complexity and pluralism can be a feature of coverage decision-making at both national and local levels. However, along a number of dimensions these phenomena tend to be more pronounced for local bodies who are: likely to be working to less clearly demarcated aims and goals; likely to be grappling with budget implications and the implementation of their decisions and; likely to have participants that are more explicitly sectional - i.e. openly advocating on behalf of their respective organisations whereas members of national bodies are more likely to be at least nominally committed to a non-partisan approach. In a UK setting, the relatively high levels of external scrutiny on national decision-makers (i.e. of the National Institute for Health and Care Excellence) also predisposes them towards the routine use of economic analysis as a means of justifying and defending their decisions (9) (Table 1).

Overall, the literature suggests a growing realisation that interventions by health economists in the area of research utilisation have not always addressed the totality of such factors, and it seems that whilst difficulties in accessing and interpreting economic studies present a continued challenge for decision-makers, issues of acceptability are more critical and far-reaching in their implications. If greater application of health economics to priority setting is the aim - and we acknowledge that this is contested - it seems logical to devise strategies which address these aspects. However, this multiplicity of factors has yet to be met with a similar scope and range of potential responses which have tended to centre on questions of how research by health economists can be made more accessible to policy-makers by increasing resources, improving the means of communication with decision-makers, and providing decision-makers with training in interpreting health economics. However, the more challenging problems of acceptability have remained relatively unaddressed and in particular it is unclear how organisational and political factors are to be addressed.

In this context, we would emphasize the importance of a sustained and rigorous examination of the congruence between economic evaluation and organisational mission and aims, links to budget flows and compatibility with performance measurement/accountability mechanisms. In this, we welcome Eckard and colleagues' attention to institutional features of decision-making bodies and believe that this should be integral to any future analysis of economic evaluation usage in priority setting. We know from other areas of study that context can shape the use of evidence in organisational and policy decision-making. If the extent to which knowledge is used in practice depends at least to some extent on institutional receptiveness, we clearly need to understand the incentives, rewards and penalties that might affect priority setters' inclination to adopt economic analysis into their decision-making (10).

At the national level there has begun in recent times to be a sustained analysis of variation between guidance producing bodies especially with regards to the terms of their delegated responsibilities, and Eckard and colleagues offer a timely intervention into this literature. We believe analysis of how these features interact with aspects of wider context to produce varying levels of demand for economic analysis would be a fruitful line of future enquiry. Much of the existing research confines its respondent group to those formally involved in the decision-making process. We believe responses from a wider range of interested parties - the public, government, clinicians and so on - might enrich future understanding of what the place of economics is, and should be, in priority setting. At the local level, where the decision-making infrastructure is most complex, future studies might also benefit from a systems oriented approach. The tendency to focus on individual decisionmaking units, whilst informative, leaves important elements of the wider environment unexamined thereby restricting the development of theories relating to the full range of determinants of behaviour. At the heart of the challenge for researchers and practitioners alike is therefore the need to more thoroughly recognise the contextual elements of the task in hand.

Overall we contend that whilst the use of cost-effectiveness information by national bodies is in large part a matter of accessibility, at local levels it requires greater consideration of context and acceptability. The impetus behind the use of economic analyses in resource allocation decisions is the requirement for greater rationality in decision processes. This arguably contributes to greater openness and transparency, and so necessitates that the information on which decisions are based is accessible to a wide audience. This accessibility imperative presents challenges to the health economics discipline and its proponents. It also suggests the need

Table 1. Institutional factors and incentives to employ cost-effectiveness analysis (9)

\begin{tabular}{lll}
\hline Institutional variable & Institutional form & Incentives to use economic analysis \\
\hline \multirow{2}{*}{ Stated aims and goals } & Explicit and relatively simple & Increased \\
& Poorly specified and/or complex and multi-dimensional & Decreased \\
Relationship to implementation & None/indirect & Increased \\
& Direct & Decreased \\
Institutional affiliation of actors & Non-sectional & Increased \\
& Sectional & Decreased \\
External scrutiny & High levels & Increased \\
& Low levels & Decreased \\
\hline
\end{tabular}


for decision-makers to embrace an open and transparent approach - something which can be difficult in healthcare where services are valued so highly and losses are so keenly contested. Rousseau (11) explores the discrepancy between what we can know (the 'evidence') and what we need to know in order to make decisions, and the inevitable gap between the two. Therefore, even where a 'rational' approach is preferred, extra work is required to translate evidence into a decision. This deliberative component of priority setting is often poorly articulated and less transparent than the evidence-generating component. Attention therefore needs to be paid to how deliberation within national guidance producing bodies might be codified and made clear to everyone. Too often there is a lack of both clarity and consensus regarding the process of moving collectively from consideration of the evidence to reaching a determination.

Overall, then it seems that despite the successes reported by Eckard and colleagues it remains at times lonely at the top for those pursuing an economics-informed approach and it is hard to avoid getting stuck in the middle where complexity and resistance is most pronounced. If progress is to be made, there is more that all parties - including from research, policy and practice settings - can do to help.

\section{Ethical issues}

Not applicable.

Competing interests

Authors declare that they have no competing interests.

Authors' contributions

Both authors have contributed equally.

Authors' affiliations

${ }^{1}$ Health Services Management Centre, University of Birmingham, Birmingham, UK. ${ }^{2}$ Centre for Clinical Epidemiology and Evaluation, University of British Columbia, Vancouver General Hospital Research Pavilion, Vancouver, British Columbia, Canada.
References

1. Eckard N, Janzon M, Levin L. Use of cost-effectiveness data in priority setting decisions: experiences from the national guidelines for heart diseases in Sweden. Int $J$ Health Policy Manag 2015; 3: 323-332. doi: 10.15171/ijhpm.2014.105

2. Williams I, Mclver S, Moore D, Bryan S. The use of economic evaluations in NHS decision making: a review and empirical investigation. Health Technology Assessment 2008; 12: 1-193. doi: 10.3310/hta12070

3. Erntoft S. Pharmaceutical priority setting and the use of economic evaluation: a systematic literature review. Value Health 2011; 14 : 587-99. doi: 10.1016/j.jval.2010.10.036

4. Silva ML, Spath HM, Perrier L, Moumjid N. The use of economic evaluation in health decision making at the macro level: a literature review. Journal de Gestion et D'Economie Medicales 2013; 31: 72. doi: 10.3917/jgem.131.0031

5. Checkland K, Harrison S, Marshall M. Is the metaphor of 'barriers to change' useful in understanding implementation? Evidence from general medical practice. J Health Serv Res Policy 2007; 12: 95-100. doi: 10.1258/135581907780279657

6. Bryan S, Williams I. Adoption of New Technologies, Using Economic Evaluation. Encyclopedia of Health Economics; 2014. p. 26-31.

7. Williams I, Bryan S. Understanding the limited impact of economic evaluation in health care resource allocation: a conceptual framework. Health Policy 2007; 80: 135-43. doi: 10.1016/j.healthpol.2006.03.006

8. Klein R. Dimensions of rationing: who should do what? BMJ 1993; 307: 309-11. doi: 10.1136/bmj.307.6899.309

9. Williams, I. Institutions and health care rationing: the example of health care coverage in the English National Health Service. Policy Polit 2013; 41: 223-9. doi: 10.1332/030557312X655477

10. Brouselle A, Lessard C. Economic evaluation to inform health care decision making: promise, pitfalls and a proposal for an alternative path. Soc Sci Med 2011; 72: 832-9. doi: 10.1016/j. socscimed.2011.01.008

11. Rousseau DM. In there such a thing as evidence-based management? Acad Manage J 2006; 31: 256-69. doi: 10.5465/ amr.2006.20208679 